\title{
JUST IN TIME OR JUST IN CASE: A CASE STUDY ON THE IMPACT OF CONTEXT IN ERP IMPLEMENTATIONS
}

\author{
D. V. Kerr \\ Faculty of Business \\ University of the Sunshine Coast \\ Luke Houghton \\ Department of International Business and Asian Studies \\ Griffith University
}

\begin{abstract}
This paper looks at a case study of an Enterprise Resource Planning System (ERP) implementation in a geographically dispersed utility company and the approach by middle management to several problem situations involving contextual issues and enduser developed (feral) systems. The paper contends that despite the extremely effective databases and sophisticated modules for business analytic functions within most ERP's, middle managers are still in the "just in case of an incident" mode of inventory management and data entered in their own feral systems may be significantly different to the inventory levels recorded in the main ERP. We contend that these problems point towards the failure of ERP systems to be context sensitive to organisations. It highlights problems in contemporary research which appears to lack understanding of different organisational contexts and how they could impact on ERP implementations. This paper argues that context and the ideals of ERP systems are often mismatched which leads to the development of feral systems, poor inventory practices and ERP systems implementation failure. The paper discusses the literature around ERP systems implementation and argues that context is an overlooked factor in the analysis of such systems. The paper uses case study evidence to demonstrate mismatched context and highlights the problems with assuming ERP systems are one size fits all. The paper concludes with a call to build more contextual research in the study of ERP implementations.
\end{abstract}

\section{INTRODUCTION}

Johns (2006) discusses the problem of context in organisational behaviour by arguing that most studies have disparate findings that appear to be disconnected. That is, studies which should be linked showing how certain factors relate to others, don't link and often have completely different findings. He notes that by changing just one contextual variable the outcome of any study is likely to change, actors responses to phenomena shifts as well as the outcome of any theory testing because context can shift the meaning of all other variables in a study. In particular, a study done by two different research teams can have totally different outcomes using the same instrument or theory 
testing device. Johns (2006) argues that context plays a very important role in determining how a theory emerges and it is often ignored in organisational behaviour research models.

In enterprise resource planning system (ERP) research and in particular the study of how large scale information systems effect organisations, there is a surprising lack of contextual analysis undertaken by researchers in the field (Quattrone and Hooper, 2001 highlight this). There are countless studies on "success factors" (Soja 2006, Remus 2007, Dawson and Owens 2008 for example) but very few if any studies on how context effects ERP implementations. As the case in this paper (discussed later), context can vary widely depending on: organisational hierarchy, structure, type of organisation (government versus private) and organisational culture. Yet, in most studies conducted in ERP implementations it is assumed that management hierarchy and organisational fit (for example) are taken as given (Tang 2007, Shanks 2005, Finney and Corbett 2007). We argue that such contextual variables should not be ignored in ERP implementations and in fact should be explained upfront to readers and practitioners alike. The main argument of this paper is that context should not be ignored in ERP implementations because as Johns (2006) notes, the slightest change of environmental factors influences the outcomes of any study (Ma 2007).

Others (Adler and Rips, 2008, Yang and Terejsen 2007) also highlight problems with context in the broader arena of research settings suggesting that factors are often localised and contextualised. This presents a problem for generalisability for example May (2007) in sociology and medicine and Dourish (2004) in computing science. It cannot be assumed that every organisation is the same (Garside, 1998, Adler and Gundersen 2008) because contextual factors such as demography, organisational type, culture and many other aspects make them different. This paper presents one study in which the context of the system mismatched the context of the organisational type and resulted in a community of practice we have called feral systems (Houghton and Kerr, 2006 and Kerr, Houghton and Burgess 2007).

This paper continues with the concept of "feral Systems" by placing it in the context of their effects on the efficiency and accuracy of the business intelligence (BI) or business analytics (BA) functions within the ERP environment of a large, geographically dispersed utility organisation. Kerr, Houghton and Burgess (2007) discussed the feral systems phenomena and provided some explanations for the reason for their development in terms of power imbalances within the organization. In particular, they have defined feral systems as "an information system [computerised] that is developed by individuals or groups of employees to help them with their work, but is not condoned by management nor is part of the corporation's accepted information technology infrastructure. Its development is designed to circumvent existing organisational information systems" (Kerr, Houghton and Burgess , 2007 page 142).

Feral systems are argued to be those mechanisms which circumvent regular systemic procedures to the extent that they create alternative means of accessing data. Previous research by the authors has found that feral systems are developed (usually by the managers themselves) to provide information to middle managers about inventory levels in a "just in case" fashion rather than the "just in time" approach advocated by many value or supply chain software modules within the ERP environment. This paper concentrates on feral systems and how their development and use with respect to reporting inventory levels could result in a different supply and demand analysis outcome. It is a contention in this paper that the inventory levels in feral systems could be vastly different to the centralized ERP levels because of the mismatched context. It is suggested that acknowledgement of these feral systems by upper management may improve the accuracy and effectiveness of BA initiatives within Utilcom, (a government owned corporation and the focal firm of the case study) and therefore create an understanding of context and how it impacts on inventory analysis. It is also contended that these (hidden inventory) levels are quite substantial and this is due to the need by middle managers of the assurance of ready accessibility to inventory items in times of an emergency. 


\section{JUST IN TIME VERSUS JUST IN CASE: MISMATCHING CONTEXTS}

Just in time management refers to the practice of keeping 'just enough' stock in inventory as is required for the day-to-day management of the enterprise (Stalk, 1998). On the other hand the just in case (JIC) approach highlights the 'just in case' practice of keeping an emergency supply 'just in case' it is required. The two are valid responses to emergent phenomena and are two different contextual factors that are diametrically opposed to each other, just in case being an 'at-hand' nonlinear emergency perspective versus just in time, being a traditional management concept about inventory practices. In particular, SAP is noted for its ability to optimise processes (Scheer and Haberman, 2000; Holstrom 2000) which could be at odds with the context of a just in case approach.

Soliman and Youssef (1998) argue, SAP is not just a tool for optimising inventory... it's a process driven 'reengineering' tool, that is often used to create new change processes (Martin and Cheung, 2000) and move an organisation towards such optimisation on much bigger scale (Larsen and Myers, 1998). Perreira (1999) also argues a similar point stating that SAP reengineering process should be used to change organisational culture towards the process optimisation built into SAP. This focus on 'process efficiency' makes assumptions about organisational structure and context (Hammer and Champy, 1993). It appears that the process minded methodology inherent in the literature and SAP also highlights an underpinning lean manufacturing ideal (Al-Mashari and Zairi, 2000).

Huang et al. (2001) argue that the lean manufacturing ideal (optimising inventory) and the ideals of SAP are linked around quality management principles that are underpinned by certain strategic assumptions about how to 'do' inventory management. SAP assumes that all organisations aspire to process efficiency and by doing this, the software naturally excludes the ideals of a just in case approach (noted in the case). Managers are often concerned about the effective operations of their section and as such like to have controls (plan B's) in place in case there is an emergency. This often leads to tensions between centralized management of inventory and the "just in case" approach many middle managers feel they need in order to give them a comfort zone for emergency situations. Such tension presents a mismatched context between what the system ideals are and what the organisational ideals are.

Due to the increasing numbers of ERP implementations and with increasing emphasis on supply chain management and customer relationship management and subsequent data warehousing, there are increasing opportunities for the BA functions within ERP systems to be used to produce forecasting and other models to help businesses to make better decisions. (Shanks. Seddon and Willcocks, 2003). For example Arnott and Pervan (2005 page 71) report that "The business intelligence (BI) movement of the late 1990s changed the direction or emphasis of EIS [executive information systems] by focusing on enterprise-wide reporting systems". The research shown this paper indicates that, with respect to inventory management and forecasting, even if the BA functions within an ERP system were used more effectively, their usefulness may not be as good as expected due, in part, to this "just in case" approach to inventory management that appears to be adopted by middle management in some organisations. In other words, the context between what we want from Enterprise wide reporting systems, and what we need 'just in case' an incident occurs can be very different.

Other research (see Locke and Lowe 2007, Wagner and Newell 2004, Parr, Shanks and Darke, 1999, for example) into ERP implementations has identified various impacts of ERP use and the authors of this paper suggest that some of these factors could be indicators that the feral systems and hence mismatched systemic contexts phenomena may also be prevalent in other companies. For example, Rikhardsson and Kræmmergaard (2006, page 36) report that a study of six Dutch companies revealed, "the impact of ES [ERP] implementation and use are seldom fully predictable by management. The 
ES [ERP] can be seen as an organizational actor in its own right; to a large extent, it influences values, culture, behaviour, processes and procedures of other actors in the organization".

This paper contends that the impact described above also affects the way employees try to cope with the changes and that it is very difficult for many employees to suddenly change their values, culture, behaviour, processes and especially their procedures for doing things. Therefore, the context of how things operate in the organisation can become threatened by the implementation of a system wide enterprise resource planning system. It is during this difficult adjustment time that the development of feral systems or the continuation of existing feral systems proliferates. The context of one overpowers and attempts to shift the other.

Other research (see Shin, Chae, Ho and Yu 2007 for example) provides some indications of employee dissatisfaction with ERP implementations and we contend that this situation of unrest, context mismatch and dissatisfaction is a breeding ground for the proliferation of unmaintained renegade feral systems. For example, although feral systems are not explicitly stated, the research reported by Rikhardsson and Kræmmergaard (2006, page 41) indicates a level of dissatisfaction amongst employees. Statements such as "All the companies mentioned that the time after going-live was characterized by user insecurity, changes, brush fires, frustration due to errors in system set-up as well as unfamiliarity with the new system" and "Some mentioned that the actual challenge was not always to get the software and hardware to work properly but to change employee behaviour and attitudes" leads to the possibility of the development of feral systems and the resultant problems with reporting and data analysis.

The research we report in this paper relates to operational management within a geographically dispersed utility company. This difference in perspectives (context) with respect to success is emphasized through the ERP implementation in this organisation. The implementation was initiated by accountants within the company and changes in accounting procedures and reporting to activity based costing were a major consideration in the decision to implement the ERP in the first place.

In other research, Gattiker and Goodhue (2004) used organisational information processing theory to determine factors that affected the local-level costs and benefits of ERP implementations. These researchers found that two organisational characteristic, namely interdependence and differentiation were related to activity coordination and information flows. Gattiker and Goodhue (2004) also determined that if differentiation between sub units was high then the organisation had to make compromises with respect to the ERP implementation. When there are different contexts in existence as is the case here (just in time versus just in case procedures), this shifts the underlying context implicit to work practices. As in many cases these are not explicitly agreed on or known, confused employees are encouraged by circumstance to develop feral systems, as demonstrated in the following case study.

The case study organisation has a high level of sub unit differentiation due to their large network infrastructure and hence provides an example of shifting context because of the decision to implement the ERP with no modifications and their culture of "just in case" inventory. Their use of inventory systems in both a just in time and just in case mode are offered as a method for understanding the different contexts (JIT versus JIC).

This study demonstrates that informal reward structures are in place that encourages JIC. The implications to organizations can be enormous from the perspective of inaccuracies in centralized data resulting in reductions in the effectiveness and accuracy of business analytic functions such as data mining and forecasting. The authors wish to highlight that the following case is not indicative of all inventory management systems and neither is it likely to be the only 'feral' system. The case is 
supplied as an example of this phenomenon in light of recent developments in the sphere of business analysis. More to the point, other feral systems could exist (and indeed the authors have highlighted this in Previous work - see Houghton and Kerr 2006, for example) in the context of this organisation. What follows is a description of inventory management practice as noted in one context. As the authors have reported elsewhere (see above) other feral systems are likely to exist in this particular organisation.

\section{THE CASE STUDY}

The research at Utilcom (a pseudonym) was qualitative in nature with interviews with key stakeholders in the supply chain being conducted. The corporation has an annual turnover of over 2 Billion dollars (AUD) and employs over 13,000 people.

Utilcom implemented the ERP, SAP R/3 in 2005. The company was one of the last to change from $\mathrm{R} / 2$ to $\mathrm{R} / 3$ due in the main to the large investment they had made in terms of modifying the $R / 2$ software to suit their own business processes. It wasn't until SAP told Utilcom that R/2 would no longer be supported that they finally decided to upgrade. Utilcom also decided to not to make the same error with respect to $\mathrm{R} / 2$, rather they implemented $\mathrm{R} / 3$ with the aim of modifying their business processes to suit the software. The implementation was designed to improve reporting and other functions including providing business intelligence capabilities. The modules involved in the implementation were: financial; material management; logistics; forecasting and planning; materials resources planning (MRP); human resources; information systems including executive information systems; project management; and office integration

\section{CASE STUDY METHODOLOGY AND FRAMEWORK}

This research uses an interpretive case study approach (Klein and Myers 1999, Walsham, 1993). The aim of this research is not to determine factors or derive an explanatory reference model, it is to gain rich insights and perspectives (Walsham, 2006). Examples of recent case work in information systems based around this approach can be found in Beverakis, Dick, and Cecez-Kecmanovic (2009), and Shaft, Teresa, Jordan L and Jasperson (2008) for example.

The main aim of this study is to gain insights into the social aspects, and specifically what role context plays, in ERP systems development. To gain these insights, the authors followed a case study approach as demonstrated in Yin (1994) and Stake (1995). For evidential sources the researchers used suggestions from the Klien and Myers (1999) framework described in Table 1:

\begin{tabular}{|l|l|}
\hline Source & Sources used in this case study \\
\hline Documentation & $\begin{array}{l}\text { Intranet postings, company annual reports and reports by the } \\
\text { information technology section of Utilcom }\end{array}$ \\
\hline $\begin{array}{l}\text { Participant } \\
\text { observation }\end{array}$ & $\begin{array}{l}\text { During the three month period at Utilcom, one of the authors } \\
\text { participated in training and conversed with personnel within Utilcom }\end{array}$ \\
\hline Interviews & The semi-structured interview approach was used \\
\hline
\end{tabular}

Table 1 - Evidence and sources for the case study.

The research project was conducted as two phases. The first was designed to develop an understanding of the workings of the supply chain and how factors such as social, technical and political factors interacted with each other in order to gauge an understanding of the social setting or context of the implementation. For this reason, 31 semi-structured interviews were undertaken with 
key stakeholders in Utilcom from a variety of levels and contexts within the organisation. Phase 2 of the research, conducted 12 months later, has 16 semi-structured interviews with identified stakeholders and key experts along the length of Utilcom's supply chain. These personnel were identified as 'expert' by the General Manager of the supply division of Utilcom and by the researchers.

\section{RESULTS}

This section is divided into the results from the three data sources shown in Table One.

\section{Documentation}

The documentation at Utilcom portrayed a positive slant to the ERP implementation and none of the publicly available documentation mentioned the use of relevant modules of SAP for BA. Intranet postings were available to all employees at Utilcom. There were no negative comments about the SAP implementation and no information was given about the possible strategic use of the system.

\section{Participant Observation}

One of the authors spent three months at the headquarters of Utilcom and this time enabled him to observe the implementation process of SAP. This period of study allowed the research team to more fully understand the underlying history of the SAP implementation. The relationships developed and observations made over the three month period at Utilcom enabled the authors to gain an appreciation of the broader context of the ERP implementation through the insights of this author's involvement. This enabled a degree of relationship building with the actors within Utilcom and allowed for a better interpretation of the meanings within the interview transcripts.

The participant in the observation is an experienced researcher with an appreciation of both qualitative and quantitative approaches to research within the Information Systems discipline. He was on academic study leave while undertaking the participant observation during this case study and had some knowledge of the problems of ERP implementations at Utilcom through his association with a general manager at Utilcom. The participant was able to attended meetings and training sessions in the organisation. He was also able to have informal discussions with staff.

The meetings covered may aspects of inventory control within the supply (shared services) division of Utilcom as well as the upcoming SAP R/3 implementation. Informal discussions related to concerns about how the help desk could handle "go live" day and the fact that many operational staff were still not trained. Other discussion points included workarounds for training and these included repeatedly hitting the return key in order to speed up the training process. This technique appeared to be adopted by many and the training sessions became a non-thinking mechanical process in order to speed up training and allow the worker to get back to work or go home as soon as possible.

\section{Interviews}

Interviews were semi-structured and open-ended in nature with questions expanding on emerging themes asked as required. The initial 31 interviews (the first stage of the research) were conducted before the implementation of the SAP R/3 and the remaining 16 were conducted a year after implementation. Coding of the relevant theme was completed by the open coding method (Nueman 2003). This involved organizing transcript passages into concepts and emerging themes by looking for a consistent pattern. Insights from this first series of 31 interviews led to the following research question: 
How effective is the current ERP approach in providing a method for employees to obtain data relevant to their own job requirements?

The Stage one interviews indicated that many of the staff at Utilcom was not fully convinced of the usefulness of the ERP implementation. This was emphasised in many occasions during both the first and second stage interviews and led to many "work-a-rounds" by staff. This identification of "worka-rounds" led the authors to the concept of "feral systems" (Kerr, Houghton and Burgess 2007).

The second stage of interviews again used the semi-structured approach and expanded on the themes identified in the first stage of interviews.

\section{Feral Systems and Business Analytics Initiatives}

As mentioned earlier, the feral systems phenomena has been established in earlier research, however the context with respect to inventory management and the possible implications to BA have not been discussed in much detail. The existence of feral systems in Utilcom was first established when middle managers were talking about what they referred to as "grass stocks". That is stocks of inventory parts that were kept in places other than warehouses for the "just in case" emergency situation. Records of these grass stocks were usually recorded on spreadsheets or databases entirely separate from the ERP system.

The case study interview transcripts revealed many cases of these grass stocks or hidden inventory. For example in the second stage of the research in response to the question "So you think that SAP has some doubtful figures?" a respondent answered

"That's what I've been led to believe from speaking to various people who are right into SAP which is an avenue I don't pursue but the figures seem to be different in a business warehouse to an SAP type figure. Queries get run and you get results, the level of confidence just isn't there." (Lower Manager - analyst)

Other comments such as "Patterns are not easy to find (no smart tool to find patterns in the data like missing inventory etc.)" and "Missing inventory because of 'under the counter' just in case and "a lot of grass stocks (hidden inventory items not shown in the central inventory)" indicate a misunderstanding or mistrust of the system and the reports it produces.

Other examples include statements by middle management (an engineer)

“... I rely heavily on we've got a diary that tracks all material usage on a daily basis so it will have on there how many [of the product] we unloaded today so Bruce will come in write in the diary in what section, how many lengths of [product] he does. That diary then goes into a database internally within here and onto a spreadsheet ...I do the inventory audits probably once every 3 to 6 months ... sort of thing.(Construction Engineer).

This statement indicates a high level of internal information systems (feral systems) are developed and used to either supplement the SAP system or replace it. In either case there is no certainty that the information will be recorded centrally in the ERP

The use of feral systems was further confirmed in Stage 2 interviews when the researcher mentioned the feral systems concept, respondents recorded statements such as;

"Yes that's a very good point and the people that make these feral systems will have no end of argument about why they are needed and yes there may be justifications but at the end of the day if everyone goes down their own little track with their own systems we are going to be in the same position as we were before we implemented R3" (Upper level manager) 
Other respondents indicated that feral systems may not be as much of a problem as indicated and that they could be confused with other reporting tools, for example;

"Certainly we've pulled data out of SAP and put it into Access databases or Excel spreadsheets to do various manipulations so to that extent yes we are doing that but I don't see them as feral systems. I see those as different reporting tools" (Upper level manager - commodities)

On the other hand the same respondent indicated that he could see problems with feral systems in certain cases where there was little or no visibility for the organisation, for example:

"At the other end of the equation there are a number of customers who are doing their planning outside of SAP so there is no visibility for the organization because their planning exists in Excel spreadsheets or access databases and isn't rolling up into any corporate plan so that does create a problem" (Upper level manager - commodities)

And:

"(Interviewer) The information from [supervisors], how does that information come? Is it stored in [enterprise] system?(Interviewee) [no] stored in email and excel. But can be referred back to. (Interviewer) Is that shared with others or not. (Interviewee) No not really.[we use] emails and telephones.

In the selected quotes above, none of the interviewees perceive a need to get information back into the main ERP system for either forecasting or knowledge management purposes.

\section{DISCUSSION}

The development of an IT strategic plan that includes the use of BA is an essential aspect in ensuring that the technology can deliver the promised strategic and competitive advantage that has been promised by much of the information systems literature. If Australian businesses wish to remain competitive in world markets, they need to adopt world's best practice with respect to the use of BA approaches.

This research may provide an insight into potential problems with BA approaches using centralized ERP modules as it appears that the "real data" maybe different to the data reported in the ERP system. If this is the case then forecasts and other BA outputs from data mining, on-line analytical processing and other explanatory or predictive models will also be inaccurate. In the case shown in this study, the 'grass stocks' have been around for quite some time and this has been reflected in forecasts over a substantial period of time. It has been estimated by the general manager of supply division that a great deal of inventory has been hidden and that Utilcom has excessive amounts of hidden stocks that are kept away from the central inventory database. The problem is that this has resulted in a continual over ordering of excessive stocks in a "just in case" mode of operation rather than the "just in time" approach that is proposed by BA as being best management practices.

Arguably, there are two failures of context in this case study that are worth noting. Firstly, there is a contextual mismatch between what is expected of the system (Just in Time) versus what happens through the culture of the organisation (Just in case). If employees wilfully hide material from the accepted system (ERP), what hope is there for a BA system to provide accurate reports if it is based on the JIT ideal? Does this kind of phenomena happen in other organisations or is it just the case in this particular organisation? Management were not aware of the feral systems that were proliferating under the ERP as operators carefully hid the JIC approach from them. Two different contexts were operating under different strategic assumptions and the effects on the ERP systems were marked. 
The lack of context arguably could be placed on the shoulders of the culture of the organisation itself and what ERPs are designed to achieve. That is, ERP systems are designed for process maximisation and efficiency. There are massive differences in processes, procedures, routines and strategic goals. While it is possible that a large percentage of the phenomena discussed in the case study is more than likely the result of a context mismatch between management and operational management ideals, it is also possible that a large amount of the context discussed here is because the ERP system ideals do not match those that are found in most public organisations.

In essence, a public institution may seek process efficiency but may be unable to due political arrangements, governmental agendas and overwhelming bureaucracy. The question for further analysis and study is: how do government owned corporations fare with the highly structured process methodology of an ERP system when in many cases the processes cannot be optimised due to multiple stakeholders and aforementioned government agendas?

As Johns (2006) argued, the slightest change can influence outcomes and in citing Gladwell's (2002) Tipping Point he notes that a handful of influential people can change the way a system behaves. Perhaps the underlying mismatch of context, JIT versus JIC, in this case is to blame for the massive project overruns and failures associated with some ERP implementations. Perhaps because of this issue, success factors may not apply in some particular contexts because they seek to generalise globally, where it might not even be possible to do so locally, within the context of a single organisation. These contextual problems are implicit, and the resulting systems development process may tease them out revealing a huge mismatch between what is being analysed and what is being developed. However, only more research in this area will be able to uncover the impact of context on ERP implementations.

\section{LIMITATIONS}

Yin (1994) notes and Eisenhardt and Graebner (2007) confirms, data can inducted from a single case for the purposes of generalisation but it's better to have a long running sample of cases to see if patterns replicate and expand the general themes found in the single case. In this case study, if there is a failure of context, then which actors, groups and sections played a role in circumventing ERP systems? Who are the people in the group that are driving the JIC paradigm? Which management strategies create the context for confusion in ERP implementations? Is a process optimisations strategy and methodology relevant given the context? This is a limitation of this study because much more needs to be known about the context and how it relates to these methodologies. A lot more work needs to be done here to establish what particular contexts effects the system in which particular way.

While this study highlights the important problem of context, it's hard to say with any degree of certainty if the results can be interpreted beyond the context shown in this particular case. In Information Systems research in this area the authors feel that much more work needs to be done in highlighting contextual variables in ERP implementations and building models to test and evaluate through case studies before too many conclusions can be drawn. Perhaps then, a discussion on the systemic effects could be worthwhile, but for now, this study only highlights several small areas where context effects the implementation of ERP systems.

\section{CONCLUSIONS}

For BA to be effective in an organisation, the fundamental information systems principles of accuracy and robust databases must be in place. Many companies spend vast amounts of money in terms of ERP implementations to ensure that their databases are accurate and robust enough to allow the 
development and use of strategic tools such as forecasting and other BA principles. However, just because the infrastructure is of a high standard, it does not ensure that the data contained in the database is accurate or contextual. This research indicates that many contextual issues in each ERP implementation need to be considered also. Issues such as rewards given to employees and mistrust of the centralized ERP have resulted in development of systems independent of the ERP. We have called these feral systems. An awareness of these systems, the social reason for their creation and the context in which they proliferate is essential for upper management to understand and consider when making strategic decisions. Complete trust in the BA coming from the ERP may lead to misleading results and errors in forecasting that may have wide ranging implications for the organisation. An appreciation of the contextual factors that lead to the development of feral systems and the resultant inaccuracies in recording true inventory levels can lead to improvements in job training as employees will be able to recognise the "object of the exercise" at more of a macro, whole of firm level.

Whilst this is not proof of the presence of feral systems in other organisations, there is certainly a great deal of anecdotal evidence to indicate the possibility in many other organisations. Other research indicates continual employee dissatisfaction with ERP implementations. This is often associated with the decision by companies not to make changes to the ERP to fit their existing business processes but a preference to change company processes to fit the ERP. These factors, as well as a reward system that still encourages the keeping of excess inventory (JIC) lead to the development of feral systems at Utilcom and we suspect the situation is similar in many other organisations.

While it is accepted that many organisations overcome initial difficulties with ERP implementations and, in time are able to take advantage of the full functionality of the system, the authors suggest that with the increasing number of mergers and acquisitions occurring in both the private and public sectors worldwide, the problem will continue well into the foreseeable future.

\section{REFERENCES}

Adler, N.J. \& Gundersen, A., (2008). International Dimensions of Organisational Behavior, Case Western Reserve University: Thomson.

Adler, J. E. and Rips, L. (eds) (2008) Reasoning: Studies of Human Inference and its Foundations, Cambridge University Press, UK.

Al-Mashari, M and Zairi, M (2000) Revisiting BPR: a holistic review of practice and development, International Journal of Physical Distribution \& Logistics Management, Vol. 6, No.1, pp. 1032.

Arnott D and Pervan G (2005) A critical analysis of decision support systems research, Journal of Information Technology, Vol. 20, pp. 67-87.

Beverakis G., Dick G. and Cecez-Kecmanovic, D (2009) Taking Information Systems Business Process Outsourcing Offshore: The Conflict of Competition and Risk. Journal of Global Information Management, Vol. 17, No. 1, pp.32-48.

Dawson, J., Owens D. (2008) Critical Success Factors in the Chartering Phase: A Case Study of an ERP Implementation, International Journal of Enterprise Information Systems, Vol. 4, No. 3, pp. 9-24.

Davenport, T.H. and Brooks, J. D. (2004) Enterprise Systems and the Supply Chain, Journal of Enterprise Information Management, Vol. 17, No. 1, pp.8-19.

Dourish, P (2004) What we talk about when we talk about context, Personal and Ubiquitous Computing, Vol. 8, No.1, pp. 19-30. 
Eisenhardt K and Graebner M (2007) Theory Building From Cases: Opportunities and Challenges, Academy of Management Review, Vol. 50, No. 1, pp. 25-32.

Finney, S. Corbett, M. (2007) ERP implementation: a compilation and analysis of critical success factors, Business Process Management Journal, Vol. 13, No. 3, pp. 329-347.

Garside, P (1998) Organisational context for quality: lessons from the fields of organisational development and change management, Quality in Health Care, Vol. 7, No. 1, pp. 8-15.

Gattiker T.F. and Goodhue D.L. (2004) Understanding the local-level costs and benefits of ERP through organizational information processing theory, Information \& Management, Vol. 41 pages $431-443$.

Gladwell, M. (2000) The Tipping Point: How little things can make a big difference, Little Brown, United States.

Hammer, M. and Champy, J. (1993), Reengineering the Corporation: A Manifesto for Business Revolution, HarperCollins, New York, NY.

Holstrom, J (1998) Business process innovation in the supply chain: a case study of implementing vendor managed inventory, European Journal of Purchasing \& Supply Management, Vol. 4, pp. 127-131.

Houghton, L and Kerr D. (2006) A study into the creation of feral information systems as a response to an ERP implementation within the supply chain of a large Government owned corporation, International Journal of Internet and Enterprise Management, Vol. 4, No. 2, pp. 135-147

Huang, S, Kwan, I and Hung, Y (2001) Planning Enterprise Resources by the use of a Reengineering Approach to build Global Logistics management system, Industrial Management and Data Systems, Vol. 101, No. 9, pp. 483-491.

Kerr D, Houghton L. and Burgess K. (2007) Power Relationships that lead to the Development of Feral Systems, Australasian Journal of Information Systems, Vol. 14, No.2, pp. 141-152.

Klein H. K. and Myers M.D. (1999) A set of principles for conducting and evaluating interpretive field studies in information systems, MIS Quarterly, Vol. 23, No.1, pp 67-94.

Larsen, M and Myers, M (1999) When success turns into failure: a package-driven business process re-engineering project in the financial services industry, Journal of Strategic Information Systems, Vol. 8, pp. 395-417.

Locke, J. and Lowe, A. (2007). A biography: Fabrications in the life of an ERP package. Organization, Vol. 14, No.6, pp 793-814.

Ma, H, (2007) Process-aware information systems: Bridging people and software through process technology, Journal of the American Society for Information Science and Technology, Vol. 58, No.3, pp. 455-456.

May C. (2007) The Clinical Encounter and the Problem of Context, Sociology, Vol. 41, No.1, pp. 2945.

Martin, I and Cheung, Y (2000) SAP and business process re-engineering, Business Process Management, Vol. 6, No. 2, pp.113-121.

Parr, A. N., G. G. Shanks, and P. Darke (1999). Identification of necessary factors for successful implementation of ERP systems. In Proceedings of the IFIP TC8 WG8.2 International Working Conference on New Information Technologies in Organizational Processes, Deventer, The Netherlands, The Netherlands, pp. 99-120. Kluwer, B.V.

Perriera R (1999) Resource view theory analysis of SAP as a source of competitive advantage in firms, ACM SIGMIS Database, Vol. 30, No. 4, pp.38-46.

Neuman, W. L. (2003) Social Research Methods: Qualitative and Quantitative Approaches, 5th Edition, Allen and Bacon, Boston, USA. 
Quattrone P and Hopper T (2001) What does organisational change mean? Speculations of a taken for granted category, Management Accounting Research, Vol 12, No. 4, p.403-435.

Remus, U. (2007) Critical success factors for implementing enterprise portals: A comparison with ERP implementations, Business Process Management Journal, Vol. 13, No. 4, pp. 538-552

Rikhardsson P and Kræmmergaard P (2006) Identifying the impacts of enterprise system implementation and use: Examples from Denmark International Journal of Accounting Information Systems, Vol. 7, pp 36- 49.

SAP (2008) Components and Tools of SAP Netweaver: SAP Netweaver business intelligence http://www.sap.com/platform/netweaver/components/bi/index.epx (accessed May 31 2008)

Scheer A and Habermann F (2000) Making ERP a Success, Communications of the ACM, Vol. 43, No. 4, pp. 57-61.

Shaft, M. Teresa, A., Jordan L., Jasperson, J (Sean) (2008) Managing change in an information systems development organization: understanding developer transitions from a structured to an object-oriented development environment, Information Systems Journal, Vol. 18, No. 3, pp.275-297.

Shanks, G., Seddon, P.B and Willcocks L (2003) Second Wave Enterprise Resource Planning Systems Implementation and Effectiveness, Cambridge University Press.

Shin, E.J, Chae Y.M., Ho SH, and Yu, K. (2007) ERP System Performance in a Hospital, Journal of Korean Society of Medical Informatics, Vol. 13, No. 2, pp. 77-82.

Soja, P (2006) Success Factors in ERP implementations, Journal of Enterprise Information Management, Vol. 19, No. 4, pp. 418-433.

Soliman, F and Youssef, M (1998) The role of SAP software in business process re-engineering, International of Operations and Production Management, Vol. 18, No. 9/10, pp. 886-895.

Stake, R. (1995). The art of case research. Thousand Oaks, CA: Sage Publications.

Stalk, G. (1998) Time: the next competitive advantage, Harvard Business Review, August, 1998.

Tang, Yinghua (2007) ERP implementation and Critical success factors: A study of Shanks ERP model on Lenovo, Nottingham University $\mathrm{PhD}$ thesis.

Wagner, E. L. and Newell, S. (2004). 'Best' for whom?: The Tension Between 'Best Practice' ERP Packages and Diverse Epistemic Cultures in a University Context, The Journal of Strategic Information Systems, Vol.13, No. 4, pp.305-328.

Walsham G., (1993) Interpreting Information Systems in Organizations, John Wiley, Chichester.

Walsham, G. (2006) Doing interpretive research. European Journal of Information Systems Vol.15, No. 3, pp.320-330.

Yang, X and Terejsen, S. (2007), In search of confidence: Context, collaboration, and constraints, Asia Pacific Journal of Management, Vol. 24, No.4, pp. 497-507.

Yin R. K. (1994) Case Study Research: Design and Methods, Sage Publications London. 